\title{
Pharyngeal Fistula, CTCAE
}

National Cancer Institute

\section{Source}

National Cancer Institute. Pharyngeal Fistula, CT CAE. NCI Thesaurus. Code C57851.

A disorder characterized by an abnormal communication between the pharynx and another organ or anatomic site. 